\title{
Imaging Strategies and Outcomes in Children Hospitalized with Cervical Lymphadenitis
}

\author{
Sanyukta Desai, MD, MSc ${ }^{1 *}$, Samir S Shah, MD, MSCE 2,3 , Matt Hall, $\mathrm{PhD}^{4}$, Troy E Richardson, $\mathrm{PhD}^{4}$, \\ Joanna E Thomson, MD, MPH' , for the Pediatric Research in Inpatient Settings (PRIS) Network.
}

'Division of Hospital Medicine, Department of Pediatrics, Seattle Children's Hospital, University of Washington School of Medicine, Seattle, Washington; 'Divisions of Hospital Medicine and of '3nfectious Diseases, Department of Pediatrics, Cincinnati Children's Hospital Medical Center, University of Cincinnati College of Medicine, Cincinnati, Ohio; ${ }^{4}$ Children's Hospital Association, Lenexa, Kansas.

OBJECTIVES: This study aimed to describe variation in imaging practices and examine the association between early imaging and outcomes in children hospitalized with cervical lymphadenitis.

METHODS: This multicenter cross-sectional study included children between two months and 18 years hospitalized with cervical lymphadenitis between 2013 and 2017. Children with complex chronic conditions, transferred from another institution, and with prior hospitalizations for lymphadenitis were excluded. To examine hospital-level variation, we calculated the proportion of children at each hospital who received any imaging study, early imaging (conducted on day 0 of hospitalization), multiple imaging studies, and CT imaging. Generalized linear or logistic mixed effects models examined the association between early imaging and outcomes (ie, multiple imaging studies, surgical drainage, 30-day readmission, and length of stay) while accounting for patient demographics, markers of illness duration and severity, and clustering by hospital.
RESULTS: Among 10,014 children with cervical lymphadenitis, $61 \%$ received early imaging. There was hospital-level variation in imaging practices. Compared with children who did not receive early imaging, children who received early imaging presented increased odds of having multiple imaging studies (adjusted odds ratio [aOR] 3.0; 95\% Cl: 2.6-3.6), surgical drainage (aOR 1.3, 95\% Cl: 1.1-1.4), and 30-day readmission for lymphadenitis (aOR 1.5, 95\% Cl: 1.2-1.9), as well as longer lengths of stay (adjusted rate ratio $1.2,95 \% \mathrm{Cl}$ : 1.1-1.2).

CONCLUSIONS: Children receiving early imaging had more resource utilization and intervention than those without early imaging. Our findings may represent a cascade effect, in which routinely conducted early imaging prompts clinicians to pursue additional testing and interventions in this population. Journal of Hospital Medicine 2020;15:197-203. ( 2020 Society of Hospital Medicine ervical lymphadenitis is a common superficial neck infection in childhood. While most children with cervical lymphadenitis recover with antibiotic therapy, a subset can develop an abscess that may require surgical drainage. Radiologic imaging, most commonly ultrasound or computed tomography (CT), is often performed to identify such an abscess. ${ }^{1-3}$ However, no national standards exist to guide clinician decision making around imaging in this population. In the absence of evidence-based guidelines, variability in frequency, timing, and modality of imaging likely exists in children hospitalized with cervical lymphadenitis.

As demonstrated for several other common pediatric conditions, $, 4,5$ variability in imaging practices may contribute to overutilization of resources in children with cervical lymphadenitis.

\footnotetext{
*Corresponding Author: Sanyukta Desai, MD; E-mail: sanyukta.desai@seattlechildrens.org; Telephone: 206-987-7370.

Published online first November 20, 2019.

Find additional supporting information in the online version of this article.
}

Received: June 25, 2019; Revised: September 18, 2019;

Accepted: September 22, 2019

๑ 2020 Society of Hospital Medicine DOI 10.12788/jhm.3333
In particular, routinely conducting imaging on presentation may constitute overuse, as children with cervical lymphadenitis who present with less than 72 hours of neck swelling rarely undergo surgical drainage within the first 24 hours of hospitalization. ${ }^{1,6,7}$ Imaging performed on presentation is often repeated later during hospitalization, particularly if the patient has not improved with antibiotic therapy. The net result may be unnecessary, redundant radiologic studies. Furthermore, serious complications such as bacteremia, extension of infection into the retropharyngeal space, or involvement of the airway or vasculature rarely occur in children with cervical lymphadenitis. $^{6.8}$ In this context, deferring initial imaging in this population is unlikely to lead to adverse outcomes and may reduce radiation exposure.

The overall objectives of this study are to describe hospital-level variation in imaging practices for pediatric cervical lymphadenitis and to examine the association between early imaging and outcomes in this population.

\section{METHODS}

\section{Study Design and Data Source}

We conducted a multicenter, cross-sectional study using the Pediatric Health Information Systems (PHIS) database, which 
contains administrative and billing data from 49 geographically diverse children's hospitals across the United States (US) affiliated with the Children's Hospital Association (Lenexa, Kansas). PHIS includes data on patient demographics, discharge diagnoses, and procedures using the International Classification of Diseases, 9th (ICD-9) and 10th Revision (ICD-10) diagnosis codes, as well as daily billed resource utilization for laboratory tests, imaging studies, and medications. Encrypted medical record numbers permit longitudinal identification of children across multiple visits to the same hospital. Use of de-identified PHIS data was deemed to be nonhuman subjects research; our approach to validation of ICD codes using local electronic medical record review was reviewed and approved by the Cincinnati Children's Hospital Medical Center Institutional Review Board.

\section{Study Population}

Our study team developed an algorithm to identify children with cervical lymphadenitis and minimize misclassification using PHIS. All children with lymphadenitis-related ICD-9 and ICD-10 discharge diagnosis codes were eligible for inclusion. Codes were validated at a single center via electronic medical record review; clinician-documented discharge diagnosis of cervical lymphadenitis or the presence of fever and unilateral or asymmetrical neck swelling with overlying skin changes was used as the reference standard. We then excluded children who did not receive antibiotics, children who received radiologic imaging not involving the head or neck (which suggested noncervical lymphadenitis or other illness), and children who had discharge diagnosis codes for other specified conditions that are sometimes associated with enlarged cervical lymph nodes but warrant different evaluation or treatment (eg, Kawasaki disease, retropharyngeal abscess, and dental abscess). Our final algorithm yielded a positive predictive value of $87.5 \%$ (95\% Cl: $79.2 \%-93.4 \%)$ when ICD9 codes were considered, and 95.1\% (95\% Cl: 88.9\%-98.4\%) when ICD-10 codes were considered.

This algorithm was subsequently applied to the PHIS database. Children ages 2 months to 18 years hospitalized at participating PHIS institutions between July 2013 and December 2017 with a diagnosis of cervical lymphadenitis as per the algorithm were eligible for inclusion. For children with multiple eligible admissions during the study period, we only included the first hospitalization. Children with complex chronic condition diagnosis codes ${ }^{9}$ were excluded, as their clinical complexity could influence decisions around timing and modality of diagnostic imaging. In addition, we excluded children who did not have an emergency department (ED) visit associated with their hospitalization. This step was intended to exclude children who were transferred from another institution, as imaging performed at outside institutions prior to transfer is not available in PHIS. To avoid overinflating hospital-level variation in the setting of a small sample size, we also excluded all children admitted to the 5 hospitals with fewer than 50 cases of cervical lymphadenitis during the study period. Our final cohort consisted of 44 PHIS hospitals.

\section{Measures of Interest}

To examine hospital-level variation in imaging practices, we measured the proportion of children at each hospital who underwent any neck imaging study, CT or ultrasound imaging, early imaging, and multiple imaging studies within a single hospitalization. Neck imaging was defined as the presence of a billing code for ultrasound, CT, or magnetic resonance imaging (MRI) study of the neck. Early imaging was defined as neck imaging conducted on day 0 of hospitalization (ie, calendar day of admission and ending at midnight). Multiple imaging studies were defined as the receipt of more than one imaging study, regardless of timing or modality. We also measured the proportion of children by hospital who received surgical drainage, defined by the presence of procedure codes for incision and drainage of abscess of the neck.

In examining patient-level association between early imaging and clinical outcomes, our primary outcome of interest was the receipt of multiple imaging studies. Secondary outcomes included rates of surgical drainage, length of stay (in hospital days), and rates of lymphadenitis-related hospital readmission within 30 days of index discharge.

\section{Covariates}

Baseline demographic characteristics included age, gender, race/ethnicity, and insurance type. We measured ED visits associated with lymphadenitis-related diagnosis codes in the 30 days prior to admission as a proxy measure for illness duration prior to presentation. To approximate illness severity, we included the following covariates: rates of intensive care unit admission on presentation, rates of receipt of intravenous (IV) analgesia on hospital days prior to surgical drainage, and rates of receipt of broad-spectrum antibiotics on day 0 or 1 of hospitalization. Broad-spectrum antibiotics were defined by an independent three-person review of available antibiotic codes (SD, SSS, and JT); differences were resolved by group consensus.

\section{Analysis}

Categorical variables were described using frequencies and percentages, while continuous data were described using median and interquartile range. We described hospital-level variation in imaging practices by calculating and comparing the proportion of children at each hospital who underwent any neck imaging study, CT imaging, ultrasound imaging, early imaging, multiple imaging studies, and surgical drainage.

Patient-level demographics and clinical characteristics were compared across groups using chi-square test. To examine the association between early imaging and outcomes, we used generalized linear or logistic mixed effects models to control for patient demographic characteristics and clinical markers of illness duration and severity, with a random effect for hospital to account for clustering. Patient demographics in the model defined a priori included age, race/ethnicity, and insurance type; clinical characteristics included prior ED visit for lymphadenitis, initial intensive care unit (ICU) admission, use of IV analgesia, and use of broad-spectrum antibiotics on day 0 or 1 of hospitalization. To assess the potential for misclassification 
related to the availability of calendar day but not time of imaging in PHIS, we conducted a secondary analysis to examine the patient-level association between early imaging and outcomes using an alternative definition for early imaging (defined as imaging conducted on day 0 or day 1 of hospitalization).

All statistical analyses were performed by using SAS version 9.4 (SAS Institute, Cary, North Carolina); $P<.05$ was considered statistically significant.

\section{RESULTS}

We identified 19,785 PHIS hospitalizations with lymphadenitis-related discharge diagnosis codes between July 1, 2013 and December 31, 2017. Applying our algorithm and exclusion criteria, we assembled a cohort of 10,014 children hospitalized with cervical lymphadenitis (Figure 1). Two-thirds of the children in our cohort were $\leq 4$ years old, $42 \%$ were non-Hispanic white, and $63 \%$ had a government payor (Table 1). Neck imaging (ultrasound, CT, or MRI) was conducted in 8,103 (81\%) children. CT imaging was performed in 4,097 (41\%) of children, and early imaging was conducted in 6,111 (61\%) of children with cervical lymphadenitis.

We noted hospital-level variation in rates of any neck imaging (median: $82.1 \%$, interquartile range [IOR]: $77.7 \%-85.5 \%$, full range: 68.7\%-93.1\%), CT imaging (median: 42.3\%, IOR: $26.7 \%$ $55.2 \%$, full range: $12.0 \%-81.5 \%$ ), early imaging (median: $64.4 \%$, IQR: 59.8\%-68.4\%, full range: $13.8 \%-76.9 \%$ ), and multiple imaging studies (median: 23.7\%, IQR: 18.6\%-28.9\%, full range: $1.2 \%-40.7 \%$; Figure 2). Rates of surgical drainage also varied by hospital (median: 35.1\%, IQR: 31.3\%-42.0\%, full range: $17.1 \%$ $54.5 \%)$

At the patient level, children who received early imaging were more likely to be $<1$ year old $(21 \%$ vs $16 \%, P<.001)$, or Hispanic or Black when compared with children who did not receive early imaging (Table 1). Children who received early imaging were more likely to have had an ED visit for lymphadenitis in the preceding 30 days ( $8 \%$ vs $6 \%, P=.001$ ). However, they were less likely to have received broad-spectrum antibiotics on admission ( $6 \%$ vs $8 \%, P<.001$; Table 1). Of the 6,111 patients who received early imaging, 2,538 (41.5\%) received CT imaging and 3,902 (63.9\%) received ultrasound imaging on day 0 . Of the 2,272 patients receiving multiple imaging studies, 116 (5.1\%) received two or more CT scans.

In multivariable analysis at the patient level, early imaging was associated with higher adjusted odds of receiving multiple imaging studies (adjusted odds ratio [aOR] 3.0, 95\% Cl: 2.6-3.6). Similarly, early imaging was associated with higher adjusted odds of surgical drainage (aOR: 1.3, 95\% Cl: 1.1-1.4), increased 30-day readmission for lymphadenitis (aOR: 1.5, 95\% $\mathrm{Cl}: 1.2-1.9)$, and longer length of stay (adjusted rate ratio: 1.2, $95 \% \mathrm{Cl}$ : 1.1-1.2; Table 2). For the subset of patients who did not receive surgical drainage during the index admission, the adjusted odds ratio for the association between early imaging at index admission and 30-day readmission was $1.7(95 \% \mathrm{Cl}$ : 1.3-2.1). About $63 \%$ of readmissions occurred within 7 days of index discharge; $89 \%$ occurred within 14 days.

In secondary analysis using an alternative definition for early imaging (ie, imaging conducted on day 0 or day 1 of hospitalization), the adjusted odds ratio for multiple imaging studies was 22.6 (95\% Cl: 15.8-32.4). The adjusted odds and rate ratios for the remaining outcomes were similar to our primary analysis.

\section{DISCUSSION}

In this large multicenter study of children with cervical lymphadenitis, we found variation in imaging practices across 44 US children's hospitals. Children with cervical lymphadenitis who underwent early imaging were more likely to receive multiple imaging studies during a single hospitalization than those who did not receive early imaging. At the patient level, early imaging was also associated with higher rates of surgical drainage, more frequent 30-day readmission, and longer lengths of stay.

To our knowledge, imaging practices in the population of children hospitalized with cervical lymphadenitis have not been previously characterized in the US; one study from Atlanta, Georgia, describes imaging practices in all children evaluated in the ED. ${ }^{1}$ Single-center studies of children hospitalized with cervical lymphadenitis have been previously conducted in Canada ${ }^{6}$ and New Zealand, ${ }^{8}$ in which $42 \%-51 \%$ of children received imaging. In our study, most (81\%) children hospitalized with lymphadenitis received some form of imaging, with $61 \%$ of all children receiving early imaging. Furthermore, $41 \%$ received CT imaging, as compared with $8 \%-10 \%$ of children in the aforementioned studies from Canada and New Zealand. ${ }^{6,8}$ This finding is consistent with a pattern of imaging overuse in the US, which has amongst the highest utilization rates globally for advanced imaging such as CT and MRI. ${ }^{10,11}$ Identifying opportunities to safely reduce routine imaging, particularly CT imaging, in this population could decrease unnecessary radiation exposure without compromising outcomes.

We also noted variability in imaging practices across PHIS hospitals. Some of this variability may be partially explained by differences in the patient population or illness severity across hospitals. However, given the absence of evidence-based best practices for children with cervical lymphadenitis, clinicians may rely on anecdotal experience or local practice culture to guide their decision making, ${ }^{12}$ leading to variability in frequency, timing, and modality of imaging.

At the patient level, we found that children who received early imaging were more likely to receive multiple imaging studies. This finding supports our hypothesis that clinicians often order a second imaging study when the initial imaging study does not clearly demonstrate an abscess, and the child subsequently fails to demonstrate clear improvement after 2448 hours of antibiotics.

Furthermore, early imaging was associated with overall increased utilization in our cohort, including increased likelihood of surgical drainage, 30-day readmission for lymphadenitis, as well as longer lengths of stay. Confounding may be one explanation for this finding. For instance, clinicians may pursue early imaging in children who present with longer duration of symptoms or more severe illness on presentation, as these factors may be associated with abscess formation. ${ }^{1,6,7}$ These 
PHIS Inpatient/Observation Discharges July 1, 2013 - Dec 31, 2017

Age 2 months -18 yrs

Lymphadentis-related diagnosis codes

$N=19,785$
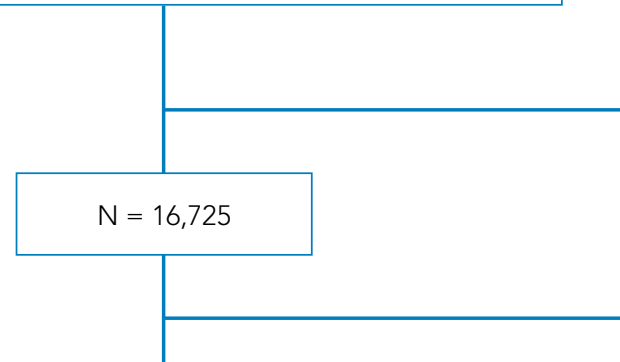

$N=12,642$

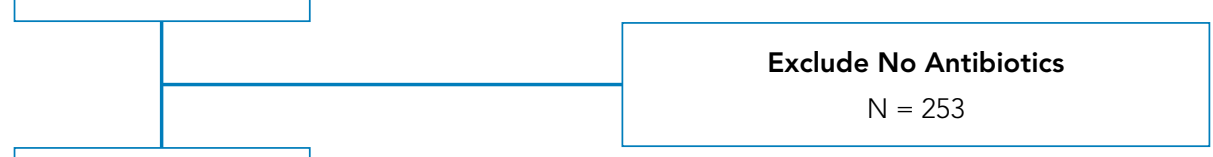

$N=12,389$

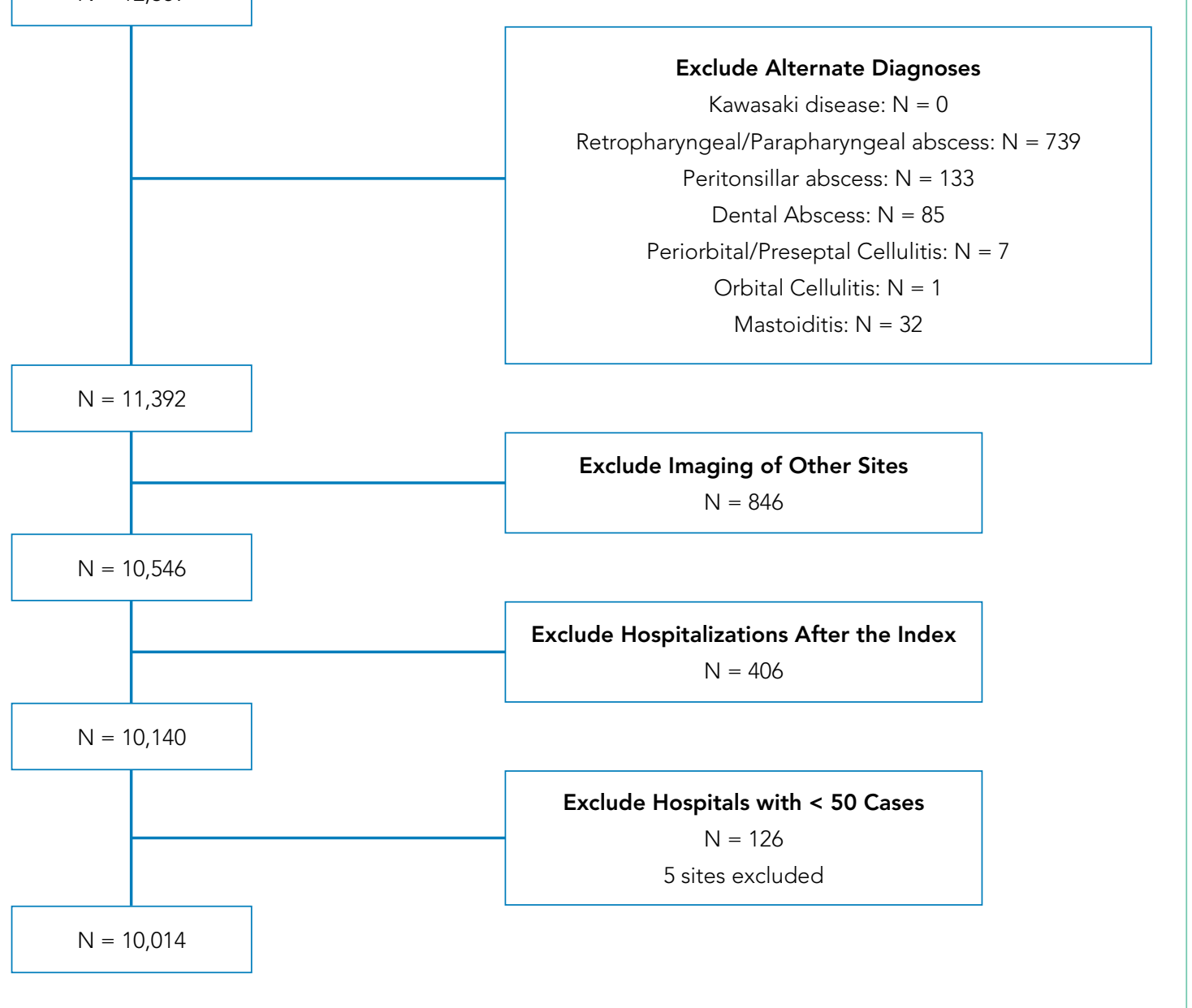

FIG 1. Patient Cohort Composition after Applying Inclusion and Exclusion Criteria

Abbreviations: PHIS, Pediatric Health Information Systems.
Exclude Complex Chronic Conditions

$N=3,060$

Exclude No ED Charge

$N=4,083$ 
TABLE 1. Baseline Demographics and Clinical Characteristics of the Study Population

\begin{tabular}{|c|c|c|c|c|c|c|c|}
\hline \multirow[b]{2}{*}{ Demographics } & \multicolumn{2}{|c|}{$\begin{array}{c}\text { Overall } \\
N=10,014\end{array}$} & \multicolumn{2}{|c|}{$\begin{array}{l}\text { No Early Imaging } \\
n=3,903\end{array}$} & \multicolumn{2}{|c|}{$\begin{array}{l}\text { Early Imaging } \\
n=6,111\end{array}$} & \multirow[t]{2}{*}{$P^{a}$} \\
\hline & $\mathrm{n}$ & $\%$ & $\mathrm{n}$ & $\%$ & $\mathrm{n}$ & $\%$ & \\
\hline$<1$ & 1,924 & (19.2) & 625 & (16) & 1,299 & $(21.3)$ & $<.001$ \\
\hline $1-4$ & 4,807 & $(48.0)$ & 1,825 & $(46.7)$ & 2,982 & (48.8) & \\
\hline $5-9$ & 1,981 & (19.8) & 830 & (21.3) & 1,151 & (18.8) & \\
\hline NonHispanic White & 4,195 & $(41.9)$ & 1,733 & $(44.4)$ & 2,462 & $(40.3)$ & $<.001$ \\
\hline NonHispanic Black & 2,316 & (23.1) & 859 & $(22.0)$ & 1,457 & (23.8) & \\
\hline Hispanic & 2,374 & (23.7) & 867 & $(22.2)$ & 1,507 & (24.7) & \\
\hline Other & 1,129 & (11.3) & 444 & (11.4) & 685 & $(11.2)$ & \\
\hline \multicolumn{8}{|l|}{ Payor } \\
\hline Prior ED visit for lymphadenitis (within 30 days of admission) & 711 & (7.1) & 236 & (6.0) & 475 & (7.8) & .001 \\
\hline Receipt of IV analgesia (preceding surgical drainage) & 446 & $(4.5)$ & 174 & $(4.5)$ & 272 & $(4.5)$ & .987 \\
\hline Broad spectrum antibiotics on day 0-1 & 659 & (6.6) & 323 & (8.3) & 336 & $(5.5)$ & $<.001$ \\
\hline Initial admission to ICU & 136 & (1.4) & 62 & (1.6) & 74 & $(1.2)$ & .111 \\
\hline \multicolumn{8}{|l|}{ All \% are column percentages } \\
\hline \multicolumn{8}{|l|}{ ap-values calculated using chi-square test. } \\
\hline Abbreviations: ED, emergency department; IV, intravenous & nsive car & & & & & & \\
\hline
\end{tabular}

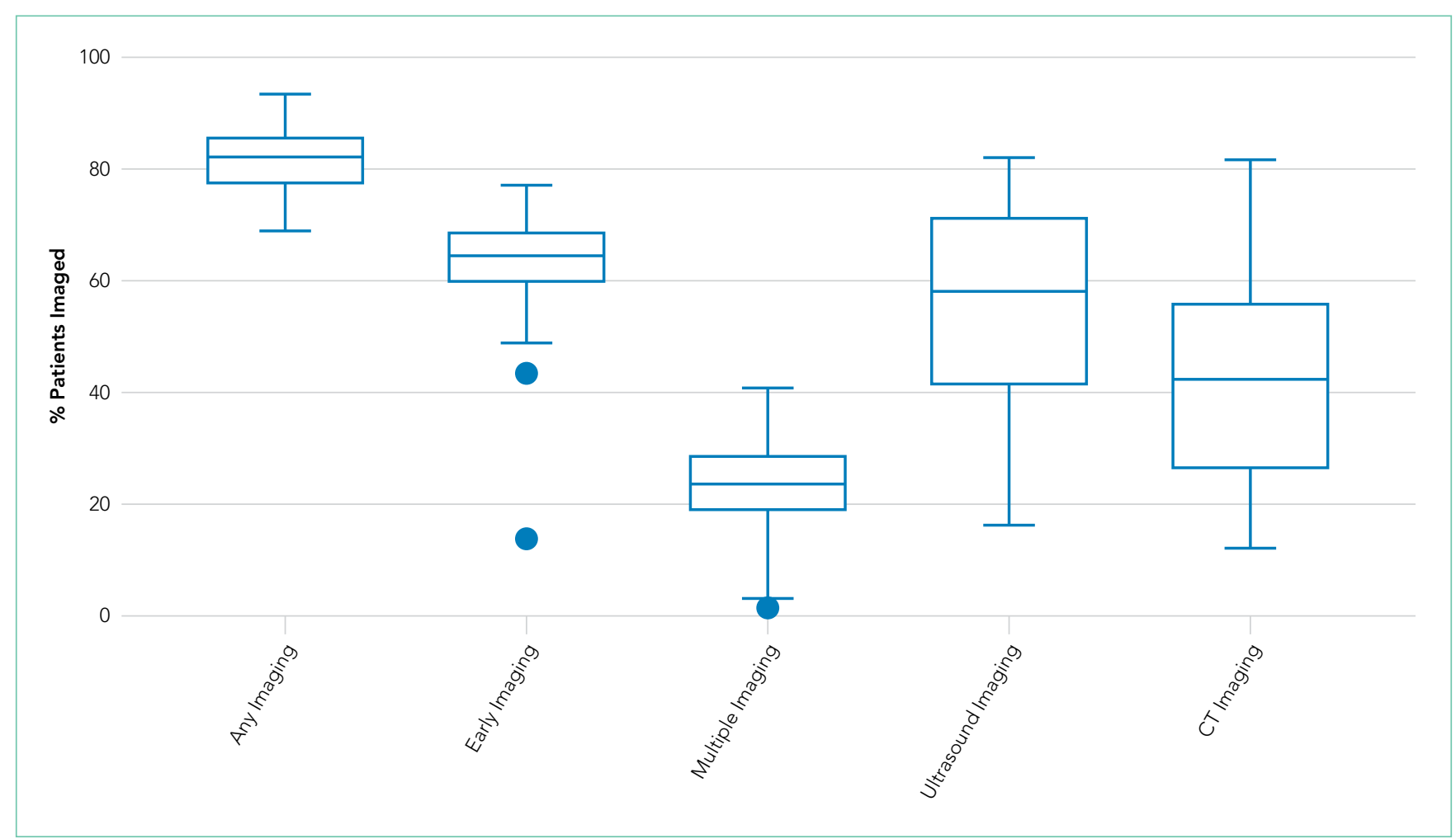

FIG 2. Hospital-level variation in imaging practices for children with cervical lymphadenitis. The line intersecting each box represents the median; the ends of the box represent the interquartile range (IQR). Whiskers represent values 1.5 times the IQR, and circles depict outliers.

NOTE: We did not include MRI imaging in the figure due to small numbers (median: $0.7 \%$ IQR: 0-1.2\%, range: 0-5.4\%) 
TABLE 2. Outcomes in Children Hospitalized with Cervical Lymphadenitis

\begin{tabular}{|c|c|c|c|c|c|c|}
\hline \multirow{2}{*}{$\begin{array}{l}\text { Outcomes } \\
\text { Multiple imaging studies }{ }^{1} \text { (\%) }\end{array}$} & \multirow{2}{*}{\multicolumn{2}{|c|}{$\begin{array}{l}\text { No Early Imaging } \\
\quad \mathrm{n}=3,903 \\
\text { Model Statistic }\end{array}$}} & \multirow{2}{*}{\multicolumn{2}{|c|}{$\begin{array}{l}\text { Early Imaging } \\
\quad n=6,111 \\
\text { Adjusted Point Estimate }(95 \% \mathrm{Cl})\end{array}$}} & \multirow{2}{*}{\multicolumn{2}{|c|}{ Modeling Results }} \\
\hline & & & & & & \\
\hline Surgical drainage $\mathrm{n}(\%)$ & 1,275 & (32.7) & 2,405 & (39.4) & Odds Ratio $(95 \% \mathrm{Cl})$ & $1.3(1.1-1.4)$ \\
\hline Length of stay (days) ${ }^{b}$ median (IQR) & 2.3 & $(2.2,2.3)$ & 2.6 & $(2.5,2.6)$ & Rate Ratio $(95 \% \mathrm{Cl})$ & $1.2(1.1-1.2)$ \\
\hline \multicolumn{7}{|c|}{ ' Generalized logistic regression with mixed effects included demographic and clinical characteristics listed in Table 1 and hospital as random effect to account for clustering. } \\
\hline \multicolumn{7}{|c|}{ ²Generalized linear mixed effects model included demographic and clinical characteristics listed in Table 1 and hospital as random effect to account for clustering. } \\
\hline \multicolumn{7}{|l|}{ Abbreviation: IQR, interquartile range. } \\
\hline
\end{tabular}

clinical covariates are not available in PHIS. Thus, we used prior ED visits for lymphadenitis to approximate illness duration, and initial admission to ICU, receipt of IV analgesia, and receipt of broad-spectrum antibiotics to approximate illness severity in an attempt to mitigate confounding. However, our proxy measures may not appropriately estimate illness duration and severity. For instance, children who had urgent care or outpatient visits for lymphadenitis would not be captured using the proxy of prior ED visit for lymphadenitis. Similarly, use of broad-spectrum antibiotics and IV analgesia may be influenced by provider or institutional preference rather than illness severity. Thus, residual confounding may exist despite adjusting for these measures.

On the other hand, it is also possible that a proportion of children with a small fluid collection on imaging may have improved with antibiotics alone. There is a growing body of evidence in children with other head and neck infections (eg, retropharyngeal abscess and orbital cellulitis with periosteal abscess) ${ }^{13-15}$ that suggests that children with small abscesses often improve with antibiotic therapy alone. In children with cervical lymphadenitis who have small or developing abscesses identified via routine imaging on presentation, clinicians may be driven to pursue a surgical intervention with uncertain benefit. Deferring routine imaging in this population may provide an opportunity to improve the value of care in children with lymphadenitis without adversely affecting outcomes.

Upon closer examination of readmissions, children who received early imaging during index hospitalization were more likely to have a 30-day readmission when only evaluating the subset of patients who did not receive surgical drainage during the index admission. This suggests that readmissions are less likely attributable to surgical complications and more likely a reflection of the natural history of lymphadenitis in which a subset of patients eventually develop an abscess. Further supporting this, $61 \%$ of children who had a 30-day readmission for lymphadenitis underwent surgical drainage during readmission. Given that lymphadenitis is a slow-brewing infection in which serious complications are rare, patients who demonstrate gradual clinical improvement do not need to remain hospitalized and serially imaged to identify a possible abscess.
Outpatient expectant management and readmission as needed for drainage may be an acceptable approach.

This study has several limitations given our use of an administrative database. Children with lymphadenitis may have been misclassified as these patients were identified using discharge diagnosis codes. To mitigate this potential misclassification, we conducted a structured validation process and found that the included codes had high positive predictive values (Appendix A). This validation process was conducted at a single hospital, and coding may vary across hospitals. To approximate sensitivity, we also sampled children without our included codes but with neck imaging and antibiotic use, and found that rates of cervical lymphadenitis were very low among children without our included diagnosis codes.

Furthermore, we were unable to measure the exact time of imaging study in PHIS; we used imaging conducted on hospital day 0 as a proxy measure for imaging conducted within the first 24 hours of presentation. With this definition, some children who had early imaging were likely misclassified as not having received early imaging. For example, a patient who arrived in the ED at 9 PM on day 0 of admission and had a neck ultrasound performed at 1 AM would be classified as having had an imaging study on day 1 of hospitalization even though the imaging study was conducted within 4 hours of presentation. Using an alternative definition of early imaging as imaging conducted on hospital day 0 and day 1 , we found a much higher adjusted OR for multiple imaging studies, with similar associations for secondary outcomes. As such, our definition of early imaging as day 0 likely biases the results toward the null; the true increase in likelihood of multiple imaging for those who receive early imaging is probably greater than our conservative estimation.

Additionally, there may be a subset of children who underwent imaging prior to presentation at the PHIS hospital ED for further workup and admission. Imaging conducted outside a PHIS hospital was not captured in this database. Similarly, children who had a readmission at a different hospital than their index admission would not be captured using PHIS. Finally, PHIS captures data from children's hospitals; practices at these hospitals may not be generalizable to practices in the community hospital setting. 


\section{CONCLUSION}

In conclusion, we found that imaging practices in children hospitalized with cervical lymphadenitis were widely variable across hospitals. Children receiving early imaging had more resource utilization and intervention when compared with children who did not receive early imaging. Our findings may represent a cascade effect, in which routinely conducted early imaging prompts clinicians to pursue more testing and interventions in this population. Future studies should obtain more detailed patient level covariates to further characterize clinical factors that may impact decisions around imaging and clinical outcomes for children with cervical lymphadenitis.

\section{Acknowledgments}

The authors would like to acknowledge the following investigators for their contributions to data interpretation and review of the final manuscript: Angela Choe MD, Cincinnati Children's Hospital Medical Center, Cincinnati, Ohio; Margaret Rush MD, Children's National Medical Center, Washington, DC; Ryosuke Takei MD, Children's Hospital of Philadelphia, Philadelphia, Pennsylvania; Wallis Molchen DO, Texas Children's Hospital, Houston, Texas; Stephanie Royer Moss MD, Cleveland Clinic, Cleveland, Ohio; Rebecca Dang MD, Lucile Packard Children's Hospital Stanford, Palo Alto, California; Joy Solano MD, Children's Mercy Hospital Kansas, Overland Park, Kansas; Nathaniel P. Goodrich MD, Children's Hospital \& Medical Center, Omaha, Nebraska; Ngozi Eboh MD, Texas Tech University Health Sciences Center, Dallas, Texas; Ashley Jenkins MD, Cincinnati Children's Hospital Medical Center, Cincinnati, Ohio; Rebecca Steuart MD, Cincinnati Children's Hospital Medical Center, Cincinnati, Ohio; Sonya Tang Girdwood MD, PhD, Cincinnati Children's Hospital Medical Center, Cincinnati, Ohio; Alissa Mclnerney MD, Maria Fareri Children's Hospital at Westchester Medical Center, Valhalla, New York; Sumeet Banker MD, MPH, New York Presbyterian Morgan Stanley Children's Hospital, New York, New York; Corrie McDaniel DO, Seattle Children's Hospital, Seattle, Washington; Christiane Lenzen MD, Rady Children's Hospital, San Diego, California; Aleisha Nabower MD, Children's Hospital \& Medical Center, Omaha, Nebraska; Waheeda Samady MD, Ann \& Robert H. Lurie Children's Hospital, Chicago, Illinois; Jennifer Chen MD, Rady Children's Hospital, San Diego, California; Marquita Genies MD, MPH, John's Hopkins Children's Center, Baltimore, Maryland; Justin Lockwood MD, Children's Hospital Colorado, Aurora, Colorado; David Synhorst MD, Children's Mercy Hospital Kansas, Overland Park, Kansas.

Disclosures: The authors have no conflicts of interest relevant to this article to disclose.

Funding: Supported by an institutional Clinical and Translational Science Award at the University Of Cincinnati College Of Medicine (National Institutes of Health National Center for Advancing Translational Sciences; 1UL1TR001425).

\section{References}

1. Sauer MW, Sharma S, Hirsh DA, et al. Acute neck infections in children: who is likely to undergo surgical drainage? Am J Emerg Med. 2013;31(6):906-909. https://doi.org/10.1016/j.ajem.2013.02.043.

2. Sethia R, Mahida JB, Subbarayan RA, et al. Evaluation of an imaging protocol using ultrasound as the primary diagnostic modality in pediatric patients with superficial soft tissue infections of the face and neck. Int J Pediatr Otorhinolaryngol. 2017;96:89-93. https://doi.org/10.1016/j.jpporl.2017.02.027.

3. Neff L, Newland JG, Sykes KJ, Selvarangan R, Wei JL. Microbiology and antimicrobial treatment of pediatric cervical lymphadenitis requiring surgical intervention. Int J Pediatr Otorhinolaryngol. 2013;77(5):817-820. https://doi. org/10.1016/j.ijporl.2013.02.018.

4. Brogan TV, Hall M, Williams DJ, et al. Variability in processes of care and outcomes among children hospitalized with community-acquired pneumonia. Pediatr Infect Dis J. 2012;31(10):1036-1041. https://doi.org/10.1097/ INF.0b013e31825f2b10.

5. Conway PH, Keren R. Factors associated with variability in outcomes for children hospitalized with urinary tract infection. J Pediatr. 2009;154(6):789-796. https://doi.org/10.1016/j.jpeds.2009.01.010.

6. Luu TM, Chevalier I, Gauthier M, et al. Acute adenitis in children: clinical course and factors predictive of surgical drainage. J Paediatr Child Health. 2005;41(5-6):273-277. https://doi.org/10.1111/j.1440-1754.2005.00610.x.

7. Golriz F, Bisset GS III, D'Amico B, et al. A clinical decision rule for the use of ultrasound in children presenting with acute inflammatory neck masses. Pediatr Rad. 2017;47(4):422-428. https://doi.org/10.1007/s00247-016-3774-9.

8. Courtney MJ, Miteff A, Mahadevan M. Management of pediatric lateral neck infections: does the adage "... never let the sun go down on undrained pus ..." hold true? Int J Pediatr Otorhinolaryngol. 2007;71(1):95-100. https://doi. org/10.1016/j.ijporl.2006.09.009.

9. Feudtner C, Feinstein JA, Zhong W, Hall M, Dai D. Pediatric complex chronic conditions classification system version 2: updated for ICD-10 and complex medical technology dependence and transplantation. BMC Pediatr. 2014;14:199. https://doi.org/10.1186/1471-2431-14-199.

10. Papanicolas I, Woskie LR, Jha AK. Health care spending in the United States and other high-income countries. JAMA. 2018;319(10):1024-1039. https:// doi.org/10.1001/jama.2018.1150.

11. Oren $O$, Kebebew E, loannidis JPA. Curbing unnecessary and wasted diagnostic imaging. JAMA. 2019;321(3):245-246. https://doi.org/10.1001/ jama.2018.20295.

12. Palmer RH, Miller MR. Methodologic challenges in developing and implementing measures of quality for child health care. Ambul Pediatr Off J Ambul Pediatr Assoc. 2001;1(1):39-52. https://doi. org/10.1367/1539-4409(2001)001<0039:MCIDAI>2.0.CO;2.

13. Daya $H$, Lo S, Papsin BC, et al. Retropharyngeal and parapharyngeal infections in children: the Toronto experience. Int J Pediatr Otorhinolaryngol. 2005;69(1):81-86. https://doi.org/10.1016/j.ijporl.2004.08.010.

14. Wong SJ, Levi J. Management of pediatric orbital cellulitis: A systematic review. Int J Pediatr Otorhinolaryngol. 2018;110:123-129. https://doi. org/10.1016/j.ijporl.2018.05.006.

15. Wong DK, Brown C, Mills N, Spielmann P, Neeff M. To drain or not to drain-management of pediatric deep neck abscesses: a case-control study. Int J Pediatr Otorhinolaryngol. 2012;76(12):1810-1813. https://doi. org/10.1016/j.ijporl.2012.09.006. 